\title{
Zur Constitution des Resacetophenons
}

von

\section{Georg Gregor.}

\begin{abstract}
Aus dem chemischen Universitätslaboratorium des Prof. R. Přibram in Czernowitz.
\end{abstract}

(Vorgelegt in der Sitzung am 20. Juni 1895.)

Die Lösung der Frage nach der Constitution des Resacetophenons scheiterte bisher an der Schwierigkeit der Feststellung des Ortes für die in das Resorcinmolekül eingetretene Acetylgruppe. Obgleich dieses Problem anscheinend leicht im Wege der Oxydation zu erledigen wäre, da zu erwarten steht, dass sich hiebei eine der drei theoretisch möglichen und auch bekannten Dioxybenzoësäuren bilden sollte, so ergaben sich bei der experimentellen Prüfung dieser Frage insoferne Schwierigkeiten, als sich überhaupt keine entsprechenden sauren Producte isoliren liessen.

Schon Nencki und Sieber, ${ }^{1}$ denen wir die Erstdarstellung des Resacetophenons verdanken, versuchten im Verfolg des Problems die Oxydation mittels Chromsäure, in der Erwartung die Acetylgruppe in eine Carboxylgruppe überführen zu können; statt der erwarteten Resorcylsäure war aber nur Essigsäure nachweisbar, ein Beweis, dass die ganze Acetylgruppe abgespalten wurde. Auch A. Wechsler ${ }^{2}$ operirte mit keinem günstigen Erfolge; sein Versuch, in alkalischer Lösung mittelst Kaliumpermanganat ausgeführt, führte ebenfalls nur zum Nachweise der Essigsäure, als des einzigen sauren Körpers, welcher sich gebildet hatte.

1 Journal fur prakt. Chemie, N. F. 23, S. 147.

2 Monatshefte für Chemie, Jahrg. 1894, S. 241. 
Dieses negative Resultat des Wechsler'schen Versuches ist umsomehr überraschend, als nach Allem, was über die Oxydation der aromatischen Methylketone mittelst Kaliumpermanganat bekannt ist, die Bildung einer Dioxybenzoësäure sicher zu erwarten stand; ja seit den umfassenden Arbeiten des Prof. Claus ${ }^{1}$ und seiner Schule, sowie der Arbeit Glücksmann's, ${ }^{2}$ dem die Oxydation des einfachsten aromatischen Methylketons, des Acetophenons, als dessen directes Analogon das Resacetophenon zu betrachten ist, gelang, war man zu der Hoffnung berechtigt, nicht nur die Dioxybenzoësäure, sondern sogar das erste Oxydationsproduct, die Dioxybenzoylameisensäure, die Ketonsäure in dem Reactionsproducte vorzufinden. Von der Voraussetzung ausgehend, dass der anormale Verlauf der Oxydation des Resacetophenons möglicherweise durch die Hydroxylgruppen des Phenols bedingt ist, glaubte ich ein passendes Derivat des Resacetophenons der Oxydation unterwerfen zu sollen. Am geeignetesten hiezu schien mir das bereits von $A$. Wechsler dargestellte Diäthylproduct. ${ }^{3}$ Der Versuch fiel durchaus günstig aus, es gelang mir nicht nur die entsprechende Ketonsäure $\mathrm{zu}$ isoliren, die als der erste bekannte Repräsentant einer Phenolketosäure zu gelten hat, sondern durch deren weitere Oxydation zu einer bekannten diäthoxylirten Resorcylsäure zu gelangen und somit die Constitution des Resacetophenons aufzuklären.

Nachstehend theile ich die Versuchsresultate mit.

\section{Oxydation des Diäthylresacetophenons. Diäthoxylbenzoyl- ameisensäure.}

Je $6 g$ reiner, in Wasser suspendirter Substanz wurden nach und nach mit einer Lösung von Kaliumpermanganat und Atzkali versetzt. Die Menge Kaliumpermanganat entsprach dem Verhältnisśe von 1 Molekül $\mathrm{C}_{12} \mathrm{H}_{16} \mathrm{O}_{3} \mathrm{zu} 2$ Molekülen $\mathrm{KMnO}_{4}$

1 Journal für prakt. Chemie, J. 1890, N. F. 41, S. 396, 483-1890, N. F. 42 , S. $508,517 .-1891$, N. F. 43 , S. 138,531 - 1891 , N. l. 44 , S. 77. 1892 , N. F. 45 , S. $377 .-1892$, N. F. 46 , S. $474 .-1893$, N. F. 47 , S. $420 .-$ Berl. Berichte, 1\&, 1856.

Monatshefte für Chemie, Jahrg. 1890, S. 246.

3 Monatshefte für Chemie, 1. c. 
und 2 Molekülen KOH. Die violette Farbe des Reactionsgemisches geht langsam ins Dunkelgrüne über und entfärbt sich innerhalb einer Woche vollkommen. Die vom entstandenen Mangandioxyd abfiltrirte Flüssigkeit wurde nun mit Äther extrahirt. Der Ätherrückstand, welcher aus unveränderter Substanz besteht, ist gering. Nach dem Ansäuern mit verdünnter Schwefelsäure bemerkt man eine Trübung, welche beim Schütteln mit Äther verschwindet.

Der Ätherrückstand ist gelblich gefärbt, sauer reagirend und erstarrt nach vollständigem Verjagen des Äthers zu einer krystallinischen Masse. Beim Umkrystallisiren aus Benzol erhielt ich den nicht scharfen Schmelzpunkt von $122-128^{\circ} \mathrm{C}$.

Zur weiteren Reinigung wurde die Säure in der Hitze in Barytwasser gelöst, das überschüssige Bariumhydroxyd durch Einleiten von Kohlensäure entfernt. Nach dem Abfiltriren vom Carbonat habe ich die das Bariumsalz enthaltende Flüssigkeit mit verdünnter Salzsäure angesäuert und mit Äther extrahirt, den Ätherrückstand aus Benzol umkrystallisirt. Die kleinen Kryställchen schmolzen scharf bei $127^{\circ} \mathrm{C}$.

Aus $60 g$ Diäthylresacetophenon erhielt ich circa $10 \mathrm{~g}$ der Saure.

Dieselbe ist unlöslich in kaltem, leichter löslich in heissem Wasser, leicht 1öslich in Alkohol, Äther, Benzol und eignet sich letzteres sehr gut zum Umkrystallisiren.

Mit verdünnter Eisenchloridlösung gibt die Säure keine Farbenreaction. In concentrirter Schwefelsäure löst sich dieselbe beim Erwärmen mit dunkelgrüner Farbe auf, die in dunkelgraublau übergebt.

Bei der Analyse ergaben sich folgende Werthe:

I. $0.2169 \mathrm{~g}$ Substanz gaben, mit Kupferoxyd verbrannt,

$0.1165 \mathrm{~g}$ Wasser und $0.481 \mathrm{~g}$ Kohlensäure.

II. $0 \cdot 2734 g$ Substanz gaben im $\mathrm{Z}$ e isel'scher Apparate $0.538 g$ Jodsilber.

In 100 Theilen:

\begin{tabular}{|c|c|c|}
\hline & Gefunden & \multirow{2}{*}{$\begin{array}{c}\text { Berechnet für } \\
\mathrm{C}_{8} \mathrm{H}_{4} \mathrm{O}_{3}\left(\mathrm{OCO}_{2} \mathrm{H}_{5}\right)_{2}\end{array}$} \\
\hline & II. & \\
\hline $.60 \cdot 4 t$ & - & $60 \cdot 50$ \\
\hline $.5 \cdot 96$ & - & $5 \cdot 88$ \\
\hline $\mathrm{OC}_{2} \mathrm{H}_{5}$ & $37 \cdot 67$ & $37 \cdot 81$ \\
\hline
\end{tabular}




\section{Oxim.}

Die vorstehend aufgestellte Formel $\mathrm{C}_{8} \mathrm{H}_{4} \mathrm{O}_{3}\left(\mathrm{OC}_{2} \mathrm{H}_{5}\right)_{2}$ fällt mit der Formel der Ketonsäure:

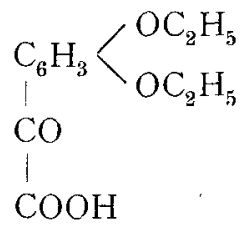

zusammen. Zum Nachweise der Ketonnatur und somit der Richtigkeit der vorskizzirten Formel stellte ich das Oxim dar. Zu diesem Zwecke wurde das Natriumsalz der Säure in Wasser gelöst, mit der fünffachen Menge salzsauren Hydroxylamins ebenfalls in wässeriger, Natriumcarbonat enthaltender Lösung versetzt. Nach einiger Zeit schied sich eine krystallinische Substanz ab, die nach mehrmaligem Umkrystallisiren aus Benzol einen Schmelzpunkt von $130^{\circ} \mathrm{C}$. (unter Zersetzung) ergab.

Da die Ausbeute der auskrystallisirten Substanz im Verhältnisse der angewandten Ketonsäure sehr klein schien, wurde das Filtrat mit Äther überschichtet und mit verdünnter Schwefelsäure angesäuert, extrahirt. Der Ätherrückstand erwies sich identisch mit dem Körper, der bei $130^{\circ}$ schmilzt.

Die Analyse des Oxims lieferte folgende Daten:

I. $0.2765 \mathrm{~g}$ Substanz gaben bei der Verbrennung mit Bleichromat und vorgelegtem Bleihyperoxyd $0.15 \mathrm{~g}$ Wasser und $0.576 \mathrm{~g}$ Kohlensäure.

II. $0.3075 g$ Substanz lieferten bei der Bestimmung nach Dumas $15 \cdot 7 \mathrm{~cm}^{3}$ feuchten Stickstoff bei $15^{\circ} \mathrm{C}$. und $737 \cdot 1 \mathrm{~mm}$ Barometerstand, entsprechend $0.01783 \mathrm{~g}$.

In 100 Theilen:

\begin{tabular}{|c|c|c|}
\hline \multicolumn{2}{|c|}{ Gefunden } & Berechnet für \\
\hline$\overline{\text { I. }}$ & TII. & $\mathrm{C}_{12} \mathrm{H}_{14} \mathrm{O}_{4}(\mathrm{~N}-\mathrm{OH}$ \\
\hline C $\ldots \ldots 56 \cdot 78$ & - & $56 \cdot 91$ \\
\hline$H \ldots \ldots 6 \cdot 02$ & - & $5 \cdot 92$ \\
\hline$N \ldots \ldots-$ & $5 \cdot 8$ & $5 \cdot 5$ \\
\hline
\end{tabular}


Nach diesen Zahlen unterliegt es keinem Zweifel, dass die erhaltene Substanz das Oxim der Ketonsäure darstellt.

Es ist demnach anzunehmen, dass die Acetylgruppe des Resacetophenons zu der Ketocarboxylgruppe oxydirt worden ist, im Sinne der Gleichung:

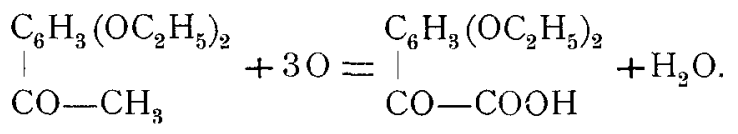

\section{Einige Salze der Ketonsäure. Silbersalz.}

Dasselbe wurde durch Erhitzen der Säure mit in Wasser suspendirtem Silberoxyd am Rückflusskühler (wobei theilweise Reduction eintrat), Filtriren und Abdampfen zur Krystallisation erhalten. Das Salz ist amorph.

Die Analyse gab folgendes Resultat:

$0.3116 g$ des Silbersalzes lieferten nach dem Glühen $0.097 \mathrm{~g}$ metallischen Silbers.

In 100 Theilen:

$$
\begin{aligned}
& \text { Berechnet } \\
& \text { Gefunden } \\
& \text { für } \mathrm{C}_{12} \mathrm{H}_{13} \mathrm{Ag} \mathrm{O}_{5} \\
& \text { Ag. . . . . . 31·12 } \\
& 31 \cdot 30
\end{aligned}
$$

\section{Natriumsalz.}

Dieses Salz wurde durch genaues Neutralisiren der Säure mit Natronlauge und Abdampfen bis zur Krystallisation erhalten. Es krystallisirt in feinen Schuppen.

$0.391 \mathrm{~g}$ der rasch zwischen Filtrirpapier lufttrocken gemachten Substanz verloren bei $120^{\circ} \mathrm{C}$. bis zur Gewichtsconstanz getrocknet $0 \cdot 1135 \mathrm{~g}$ an Gewicht.

Der Rückstand mit concentrirter Schwefelsäure abgeraucht und geglüht gab $0.0758 \mathrm{~g}$ Natriumsulfat.

In 100 Theilen:

$$
\begin{aligned}
& \text { Berechnet für } \\
& \text { Gefunden } \\
& \mathrm{H}_{2} \mathrm{O} \ldots .29 \cdot 02 \\
& \underbrace{\mathrm{C}_{12} \mathrm{H}_{13} \mathrm{NaO}_{5}+6 \mathrm{H}_{2} \mathrm{O}} \\
& 29 \cdot 34 \\
& \mathrm{Na} \ldots . .6 \cdot 6 \cdot 27 \\
& 6 \cdot 25
\end{aligned}
$$




\section{Bariumsalz.}

Die Säure wurde in Barytwasser gelöst, das überschüssige Bariumhydroxyd durch Einleiten von Kohlensäure entfernt und das Filtrat bis zur Krystallisation abgedampft. Es scheidet sich das Salz in kleinen Kryställchen ab.

$0.209 \mathrm{~g}$ der rasch zwischen Filtrirpapier lufttrocken gemachten Substanz verloren bei $120^{\circ} \mathrm{C}$. bis zur Gewichtsconstanz getrocknet $0.0395 g$ an Gewicht.

Der Rückstand mit concentrirter Schwefelsäure abgeraucht und geglüht gab $0 \cdot 06+g$ Bariumsulfat.

In 100 Theilen:

$$
\begin{array}{cc}
\mathrm{H}_{2} \mathrm{O} \ldots \ldots \ldots 18 \cdot 89 \\
\mathrm{Ba} \ldots \ldots \ldots 17 \cdot 99
\end{array} \quad \begin{gathered}
\begin{array}{c}
\text { Berechnet für } \\
\left(\mathrm{C}_{12} \mathrm{H}_{13} \mathrm{O}_{5}\right)_{3} \mathrm{Ba}+8 \mathrm{H}_{2} \mathrm{O}
\end{array} \\
19 \cdot 07 \\
18 \cdot 14
\end{gathered}
$$

Andere Salze wurden dargestellt, indem das Ammonsalz der Ketonsäure in wässeriger Lösung mit den Acetaten einiger Metalle versetzt wurde.

Quecksilberacetat erzeugt einen starken weissen Niederschlag.

Bleiacetat erzeugt einen weissen Niederschlag.

Kupferacetat erzeugt in concentrirter Lösung einen grünlichweissen Niederschlag.

Zinkacetat erzeugt ebenfalls in concentrirter Lösung einen weissen Niederschlag.

\section{Reduction der Ketonsäure.}

$1 g$ der Ketonsaure wurde in Wasser unter Zusatz von etwas Kalilauge gelöst und mit $20 \mathrm{~g}$ Natriumamalgam $(4 \%)$ versetzt. Die Mengenverhältnisse entsprachen dem Verhältnisse von 1 Molekül Ketonsäure zu 2 Molekülen Natrium, von letzterem vorsichtshalber die vierfache Menge. Nach eintägigem Stehenbleiben wurde abfiltrirt, mit verdünnter Schwefelsäure angesäuert und mit Äther extrahirt. Der nach dem Abdestilliren des $\ddot{A}$ thers verbleibende Rückstand ist schwach gelblich gefärbt 
und zeigt nach dem Umkrystallisiren aus Benzol den scharfen Schmelzpunkt von $116^{\circ} \mathrm{C}$.

Die Ausbeute ist nahezu eine theoretische. Die Säure löst sich beim Erwärmen in Benzol auf und scheidet sich beim Erkalten des letzteren, in kleinen Kryställchen ab. Das Benzol eignet sich sehr gut zum Umkrystallisiren. Die Säure ist ziemlich leicht in heissem Wasser löslich, dessgleichen in Alkohol und Äther. Auf Zusatz von wässeriger Eisenchloridlösung entsteht keine Farbenreaction. In concentrirter Schwefelsäure löst sie sich mit dunkelblauvioletter Farbe auf, auf Zusatz von Wasse scheidet sich ein Körper in violetten Flocken ab, die sich in Kalilauge farblos auflösen.

Mit Hydroxylamin reagirt die Säure nicht. Da ich bei der Elementaranalyse keine auf eine zu erwartende Alkoholsäure stimmenden Zahlen erhielt, versuchte ich die Substanz von etwa nebenbei bei der Reduction entstandener indifferenter Substanzen zu befreien.

Ich löste desshalb die Säure in Natriumcarbonatlösung auf und schüttelte diese mit Äther. Ein Ätherrückstand, der aus indifferenter Substanz besteht, ist zwar erhalten worden, doch war derselbe zu gering, als dass er näher identificirt werden konnte, und sein Vorhandensein dürfte die Ursache des vorerwähnten Mangels an guter Übereinstimmung der Analysen gewesen sein.

Die aus dem Natriumsalz durch Ansảuern mit verdünnter Schwefelsäure und Schütteln mit Äther freigemachte und durch mehrmaliges Umkrystallisiren aus Benzol ganz rein erhaltene Säure schmolz bei $115^{\circ} \mathrm{C}$. und gab bei der Elementaranalyse auf eine Alkoholsäure stimmende Zahlen.

Ich erhielt folgende analytische Daten:

$0.2588 \mathrm{~g}$ Substanz gaben mit Kupferoxyd verbrannt $0.162 \mathrm{~g}$ Wasser und $0.5667 g$ Kohlensäure.

In 100 Theilen:

$$
\begin{aligned}
& \text { Berechnet } \\
& \text { Gefunden fur } \mathrm{C}_{12} \mathrm{H}_{1 f^{3}} \mathrm{O}_{5} \\
& \text { C .... 59.73 } 60 \cdot 00 \\
& \mathrm{H} \ldots \ldots .6 \cdot 95 \quad 6 \cdot 66
\end{aligned}
$$




\section{Silbersalz.}

Dasselbe wurde durch Kochen der Säure in wässeriger Lösung mit Silberoxyd am Rückflusskühler, Abfiltriren und Eindampfen dargestellt, wobei theilweise Reduction eintrat.

Bei der Analyse erhielt ich folgendes Resultat:

$0 \cdot 3258 g$ des Salzes gaben beim Glühen $0 \cdot 102 g$ Silber.

In 100 Theilen:

$$
\mathrm{Ag} \ldots \ldots \ldots+31 \cdot 30 \quad \underbrace{\text { Gefunden }}_{31 \cdot 12}
$$

Andere Salze wurden dargestellt, indem mässig concentrirte Lösung des Ammonsalzes mit mässig concentrirter Lösung von Acetaten einiger Metalle versetzt wurde.

Calcium-, Barium - und Kupferacetat erzeugen keinen Niederschlag; Bleiacetat erzeugt einen weissen gelatinösen, Mercuroacetat einen starken weissen Niederschlag.

\section{Oxydation der Ketonsäure.}

Es stand zu erwarten, dass die Ketocarboxylgruppe durch Oxydation in eine Carboxylgruppe sich werde überführen lassen und dass es hiemit möglich sein werde, zu einer der drei bekannten diäthylirten Resorcylsäuren zu gelangen.

$2 g$ der Ketonsäure wurden mit der entsprechenden Menge Kaliumpyrochromat und Schwefelsäure in wässeriger Lösung durch circa 2 Stunden am Rückflusskühler erwärmt und hierauf längere Zeit stehen gelassen. Die dunkelgrün gefärbte Flüssigkeit wurde mit Äther extrahirt, der Ätherrückstand mit Kalilauge neutralisirt, die Lösung mit Thierkohle gekocht, filtrirt und mit Bariumchloridlösung versetzt. Der entstandene geringe Niederschlag, der nur von einer neugebildeten Säure herrühren konnte, da das Bariumsalz der Ketonsäure löslich ist, war braun gefärbt. Derselbe wurde mit Wasser aufgeschwemmt, mit Salzsäure angesäuert und mit Äther extrahirt. Der Ätherrückstand war gelblich gefärbt, harzartig. Derselbe wurde wieder mit Kalilauge neutralisirt, in wässeriger Lösung mit 
Thierkohle behufs Entfärbung gekocht, abermals mit Bariumchloridlösung gefällt und wie früher die Säure gewonnen. Durch dieses wiederholte Reinigen der ohnehin kleinen Menge verringerte sich dieselbe so, dass man weitere Versuche nicht mehr anstellen konnte. Man muss annehmen, dass eine Oxydation mit Chromsäurelösung eine tiefer gehende Zersetzung des ganzen Moleküls zur Folge hat.

Ich versuchte daher die Ketonsäure mit Kaliumpermanganat in schwefelsaurer Lösung zu oxydiren.

Je $0.50 \mathrm{~g}$ Ketonsäure wurden mit Wasser erhitzt und unter Zusatz von verdünnter Schwefelsäure mit einer Permanganatlösung, welche $0.15 \mathrm{~g}$ Kaliumpermanganat enthielt, partienweise versetzt. Die Menge Kaliumpermanganat entsprach dem Verhältniss von 5 Molekülen Ketonsäure zu 2 Molekülen Kaliumpermanganat.

Nach dem Abkühlen wurde mit Äther extiahirt, der Ätherrückstand mit verdünnter wässeriger Kalilauge neutralisirt und mit Bariumchloridlösung gefällt, das entstandene Bariumsalz in Wasser aufgenommen, mit Salzsäure angesäuert und wieder mit Äther extrahirt. Der Rückstand ist zwar gering, doch ist die Ausbeute besser als beim Oxydiren mit Chromsäure.

Bei wiederholter Darstellung in der beschriebenen Art erhielt ich genügend viel, um die Säure zu identificiren.

Der Ätherrückstand ist gelblich gefärbt; derselbe wurde in verdünntem Ammoniak gelöst, diese Lösung behufs Entfärbung mit Thierkohle gekocht, filtrirt und nach dem Erkalten mit verdünnter Salzsäure angesäuert. Der entstandene flockige Niederschlag wurde mit destillirtem Wasser ausgewaschen, getrocknet, in Benzol gelöst. Die Benzollösung mehreremal mit Thierkohle gekocht, filtrirt und zur Verdunstung des Lösungsmittels stehen gelassen. Man erhält so die Substanz in feinen weissen, seideglänzenden Schuppen, die bei $99^{\circ} \mathrm{C}$. scharf schmelzen.

Die Säure reagirt nicht mit wässeriger Eisenchloridlösung.

Bei der Analyse erhielt ich folgende Werthe:

I. $0 \cdot 2561 \mathrm{~g}$ Substanz gaben mit Kupferoxyd verbrannt $0 \cdot 154 \mathrm{~g}$ Wasser und $0.5871 \mathrm{~g}$ Kohlensäure. 
II. $0 \cdot 2615 g$ Substanz gaben im Z eisel'schen Methoxylapparate $0 \cdot 5801 \mathrm{~g}$ Jodsilber.

In 100 Theilen:

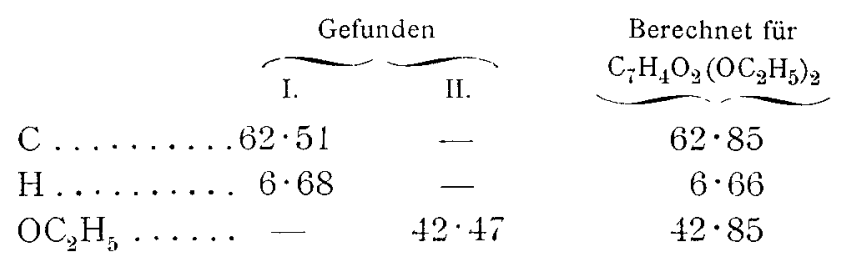

\section{Silbersalz.}

Dasselbe wurde durch Fällen des Ammonsalzes mit Silbernitratlösung erhalten. Bei der Analyse ergab sich nachstehendes Resultat:

$0 \cdot 3218 g$ desselben gaben beim Glühen $0 \cdot 109 g$ metallischen Silbers.

In 100 Theilen:

$$
A g \ldots \ldots+33 \cdot 87 \quad \frac{\underbrace{\text { Berechnet }}_{\text {Gefunden }}}{34 \cdot 06}
$$

Vorstehende Analysen beweisen, dass die Ketonsaure durch Oxydation in eine diäthylirte Resorcylsäure übergeführt wurde und dass letztere - nach dem Schmelzpunkte zu schliessen - identisch ist mit der durch Oxydation des diäthylirten Resorcylaldehyds von Tiemann und Le w $\mathrm{y}^{1}$ gewonnenen unsymmetrischen Diathoxylresorcylsäure:

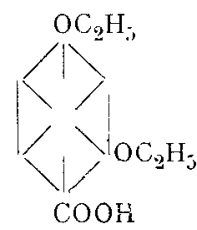

Dem Resacetophenon glaube ich demnach auf Grund vorstehender Thatsachen die Constitutionsformel:

1 Berichte der deutschen chem. Gesellsch., 10, 2215. 
<smiles>CC(=O)C12C3CC1(OC3=O)C2(O)O</smiles>

beilegen zu dürfen, umsomehr als eine Atomumlagerung innerhalb des Resorcinmoleküls bei den stattgehabten Reactionen wenig Wahrscheinlichkeit für sich hat. Diese Stellung für das eingetretene Acetyl steht auch sonst mit anderweitigen Erfahrungen im besten Einklang, da das zugehörige Wasserstoffatom am leichtesten substituirbar ist. Ich erinnere in dieser Hinsicht an die Substitution des in Rede stehenden Wasserstoffatoms durch $\mathrm{COOH}$ (Bistrzycki und Kostanecki ${ }^{1}$ ), durch CSSH (Přibram und Glücksmann ${ }^{2}$ ), durch COH (Tiemann und Lew $\left.{ }^{3}\right)$ etc.

Wenn nun auch die Beweisführung für die Constitution des Resacetophenons als ausreichend gelten muss, so versuchte ich dennoch zur Ergänzung und weiteren Bestätigung der Verhältnisse die unsymmetrische Resorcylsäure selbst zu äthyliren, in der Absicht, dadurch abermals $z u$ der oben beschriebenen diäthylirten Säure zu gelangen.

Die in guter Ausbeute isolirte Säure erwies sich aber nach dem gewichtsanalytischen Befunde als eine Monoäthylsäure, deren zweite Hydroxylgruppe wahrscheinlich eine Umlagerung zur Carbonylgruppe erfahren hat. Ich gedenke in einer nächsten Publication über diese interessante Verbindung, sowie auch über die anderen sich gleichzeitig gebildeten Producte der Reaction, mit deren Studium ich gegenwärtig beschäftigt bin, ausführlich zu berichten.

I Berichte der deutschen chem. Gesellsch., 18, 1984 und 3202.

2 Monatshefte, 1892, S. 623.

3 Berichte der deutschen chem. Gesellsch., 10, 2212. 